Case Report

\title{
Anesthetic Considerations in Bilateral Congenital Anophthalmia: A Rare Clinical Entity
}

\author{
Lalit Gupta, DA, DNB, MNAMS*; Manisha Manohar, MD \\ Department of Anesthesia, Maulana Azad Medical College \& Associated Hospital, New Delhi, India \\ "Corresponding author \\ Lalit Gupta, DA, DNB, MNAMS \\ Assistant Professor, Department of Anesthesia, Maulana Azad Medical College \& Associated Hospital, New Delhi, India; E-mail: $\underline{\text { alit.doc@gmail.com }}$
}

Article information

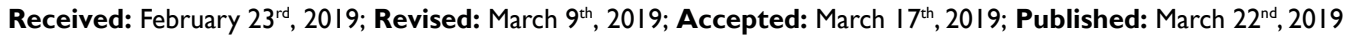

\section{Cite this article}

Gupta L, Manohar M. Anesthetic considerations in bilateral congenital anophthalmia:A rare clinical entity. Res Pract Anesthesiol Open J. $2019 ; 4(\mathrm{I}): 4-6$. doi: 10.17|40/RPAOJ-4-I22

\begin{abstract} planned for excision of right ocular swelling.

Keywords

Ophthalmopathies; Congenital anophthalmia; Microophthalmia; Ocular surgery.

\section{INTRODUCTION}

A nophthalmia is the absence of ocular tissue within the orbits. IIt represents a phenotypic continuum with microphthalmia in which the eyes are rudimentary or hypoplastic. Very often clinical anophthalmia may prove to be severe microphthalmia on imaging. With an estimated incidence of 3 per 10000 live births, it is one of the rare congenital anomalies that can present as an isolated finding or as a part of the syndrome. ${ }^{1,2}$ Genetic, as well as environmental factors have been implicated as the cause of anophthalmos. Advanced maternal age, low birth weight, infections like toxoplasmosis, rubella, cytomegalovirus, varicella virus, influenza virus, and parvovirus have been implicated.
\end{abstract}

Congenital anomalies planned for ocular surgeries range from the rare to atypical to common. Many of this rare ophthalmopathy are associated with clinical syndromes and have important anesthetic implications. Not only is it important to know the syndrome we are dealing with, but it's also the more important to understand the systems that are involved, the extent of involvement, potential anesthetic complications, right from the cerebrovascular, cardiovascular, endocrine, metabolic, neuromuscular, genitourinary systems to airway. Understanding these aspects becomes more important in rare clinical scenarios as it helps to plan the case, anticipate and treat the complications. Congenital anophthalmia is one of the rare conditions with an incidence of $<3 / 1000$ with microphthalmia reported in up to $11 \%$ of blind children, hence we report a rare case of bilateral congenital anophthalmia
Anophthalmos can present as a unilateral or bilateral lesion and can be associated with other systemic malformations particularly involving the cardiac, musculoskeletal and central nervous system (CNS). Cardiac associations include atrial septal defect (ASD), ventricular septal defect (VSD), and tetralogy of fallot, hypoplastic left ventricle, pulmonary valve atresia, and bicuspid aortic valve. ${ }^{4}$ Bilateral anophthalmia has a high rate of associated
CNS abnormalities which include septo-optic dysplasia, corpus callosum dysgenesis, absence of the anterior pituitary, agenesis of corpus callosum, absence of septum pellucidum, dilatation of the ventricles, polymicrogyria. Because of the absence of the normal globe the growth of the orbital cavity, intraorbital soft tissue, maxilla, maxillary sinus and mandible is also affected leading to facial asymmetry especially in bilateral cases where sunken orbit and midfacial hypoplasia is typical. ${ }^{5-7}$

Once a clinical diagnosis is made ocular imaging including ultrasound, computed tomography (CT), and magnetic resonance imaging (MRI) are done to establish the diagnosis. Though a clinical diagnosis, anophthalmos can prove to be microphthalmos on imaging or after surgical exploration.

\section{CASE REPORT}

A 33-year-old male patient with a history of absent eyeballs at birth presented with an insidious onset, gradually progressive painless soft globular swelling in the right eye over a period of four months. His maternal history regarding maternal age and any exposure to 
drugs, infection or environmental toxins during pregnancy was not available as his mother was not alive. On local examination the mass was soft, approximately $4.5 \times 4.5 \mathrm{~cm}$, cystic in consistency. General physical examination revealed no clinically significant abnormality with stable vitals of pulse $90 /$ minute, regular with BP of 124/84 mm-hg and BMI of $24.5 \mathrm{~kg} / \mathrm{m}^{2}$. Auscultation of heart revealed normal heart sounds and no murmurs. Auscultation of heart revealed normal heart sounds and no murmurs. Airway examination revealed bearded patient with a beard, Mallampatti grade 3 , the normal range of neck extension and flexion. All routine hematological and biochemical investigations were within normal limits. Chest radiograph and ECG were unremarkable. An outside done echocardiography report revealed trivial tricuspid regurgitation with a perimembranous VSD of $2.7 \mathrm{~mm}$ with ejection fraction of $40-45 \%$ and right ventricular pressure of $18 / 7 \mathrm{mmHg}$.

MRI reported a large lobulated well defined cystic lesion measuring $6.1 \times 4.2 \times 4.1 \mathrm{~cm}$ in the intrazonal and extraconal compartments of the right orbit causing expansion of the bony orbit and displacing the medial and lateral rectus muscles. Inferiorly, the lesion was seen to cause the indentation and downward displacement on the roof of the right maxillary sinus. Enhancing oval soft tissue was seen medial to the above-mentioned lesion suggestive of calcification likely rudimentary ocular globe with the hypoplastic right optic nerve. Similar small lesions were seen in the anteroinferior aspect of the extraconal compartment of the left orbit. All the intracranial structures were normal.

After obtaining written informed consent, the patient was taken to the operating room. A $20 \mathrm{G}$ intravenous cannula was secured on the right hand. All the standard anesthesia monitors were attached. Difficult bag-mask ventilation was anticipated due to the presence of beard so preoxygenation was done for three minutes using an rendell-baker-soucek (RBS) mask in order to ensure that no external pressure is exerted on the ocular swelling (Figure 1 and 2). Induction was done with Fentanyl $2 \mu \mathrm{g} / \mathrm{kg}$, Propofol $2 \mathrm{mg} / \mathrm{kg}$ and Vecuronium $0.1 \mathrm{mg} / \mathrm{kg}$. The airway was secured using a pro-seal laryngeal mask airway no 4 after ensuring adequate bilateral chest expansion and square wave end-tidal carbon dioxide. The device was secured in place and the patient was shifted to pressure control mode of ventilation. Anesthesia was maintained with oxygen, nitrous oxide $\left(\mathrm{N}_{2} \mathrm{O}\right)$, and isoflurane. Intravenous paracetamol and diclofenac were given as a part of multimodal analgesia. The patient developed an episode of bradycardia $\mathrm{HR}=45 / \mathrm{min}$ at the time of dissection of medial rectus muscle following which the surgeon was informed and the traction on the medial rectus was released. Local anesthetic proparacaine drops ( 2 drops) were instilled in the surgical field and the surgeons were told to proceed after 15 seconds. On exposure of the lateral rectus muscled the procaine drops were again instilled in the surgical field. Henceforth, the patient remained stable with no hemodynamic disturbances. Towards the end of anesthesia, ondansetron $4 \mathrm{mg}$ was administered. The patient was reversed with neostigmine and glycopyrrolate.
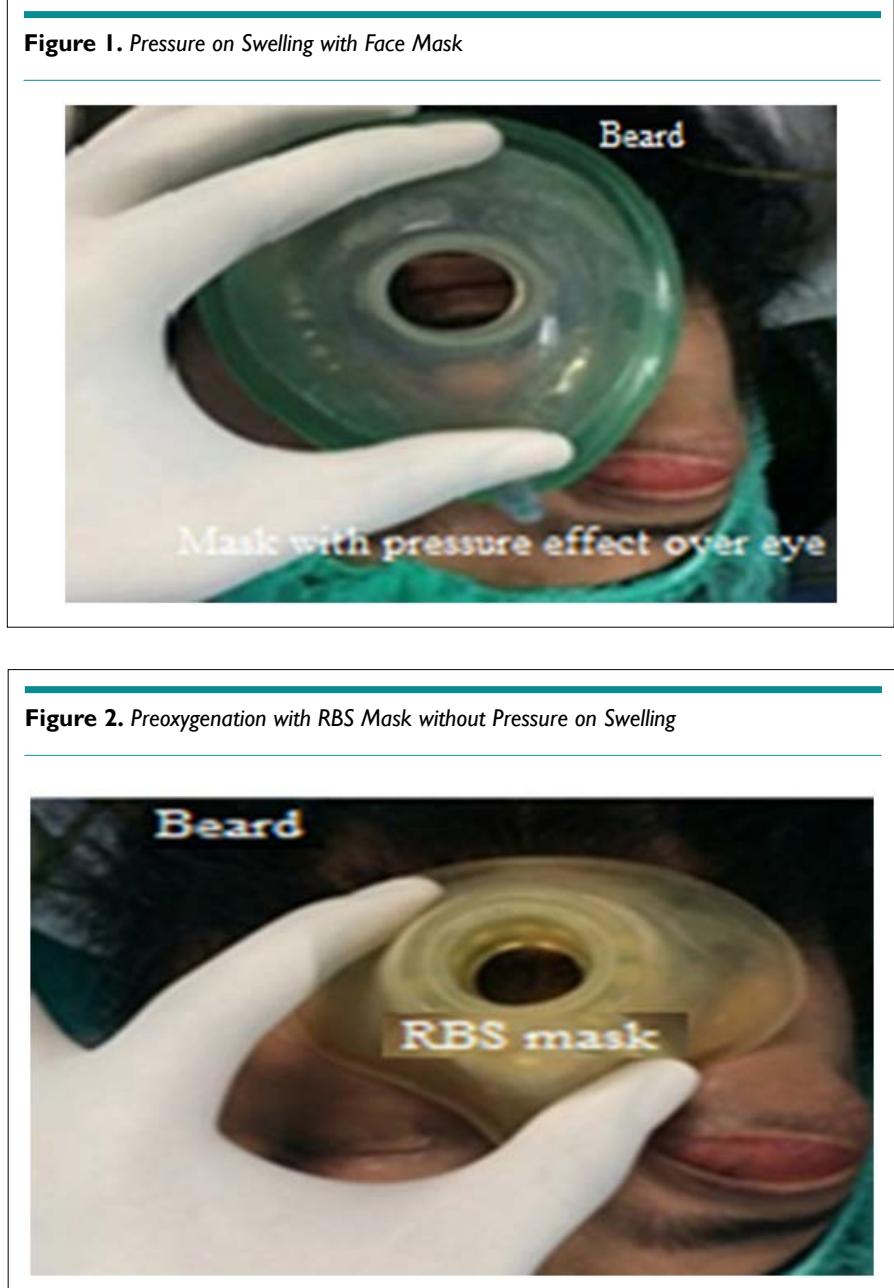

\section{DISCUSSION}

An anophthalmic pediatric or an adult patient may be planned for any kind of elective or emergent surgical procedure. A detailed birth history, documentation of any significant perinatal events, family history of the similar disease can give an important clue regarding the aetiology. Toxoplasmosis, Other Agents, Rubella or German Measles, CMV: Cytomegalovirus, and TORCH: Herpes Simplex ggroup of infections and concomitant associations of TORCH need to be ruled out in pediatric age group. Many syndromes and malformations (e.g., anophthalmia-oesophageal-genital syndrome, Matthew-Wood syndrome, CHARGE syndrome, oculo-facialcardio-dental-syndome, heterotaxy, and Fraser syndrome) have been associated with anophthalmia. A detailed clinical examination with special emphasis on the cardiac, central nervous system, and facial anatomy should be performed. A difficult airway may be anticipated in patients with facial asymmetry. Depending on the clinical findings, patients may require pre-operative echocardiography, magnetic resonance imaging of the brain and ultrasonography of the abdomen to rule out any cardiac, central nervous system or abdominal pathology, respectively. This may further help in modifying the anesthesia plan, choosing appropriate airway management device, induction, and maintenance agents while keeping in consideration the associated malformations and 
organs involvement.

Our patient was planned for excision of the ocular mass. Pre-operative MRI ruled out an intracranial extension of the mass and associated cerebral lesions. A pre-operative ECHO suggested the presence of trivial tricuspid regurgitation with a VSD of 2.7 $\mathrm{mm}$ and ejection fraction (EF) 40-45\%. Due to the presence of beard, we anticipated difficult bag-mask ventilation and a possibility that keeping a tight seal facemask can exert further pressure on the ocular mass, which may even lead to bleeding or pressure effects (raised intraocular pressure, which can lead to bradycardia). In order to avoid any external pressure on the ocular swelling an RBS mask (Figure 2) was used for pre-oxygenation.

VSD as in our case can occur as an isolated defect or as a component of a combination of anomalies. Only small or moderate-sized defects are seen as asymptomatic finding in adulthood, as most of the patients with large defects present early in life. The balance between systemic vascular resistance (SVR) and pulmonary vascular resistance (PVR) is of primary importance under anesthesia to reduce the shunt fraction and hence chances of reversal of shunt leading to further complications. VSD being a left-to-right shunt tends to increase with increase in SVR and decrease in PVR, thus leading to increased pulmonary blood flow, pulmonary hypertension, and consequently reversal of the shunt. Thus, in order to avoid these complications, SVR should be decreased by maintenance of good hypotensive anaesthesia as we did in this case. This was also the reason to prefer LMA ProSeal $^{\mathrm{TM}}$ over the endotracheal tube for avoiding increase in Systemic pressures associated with laryngoscopy and intubation.

Due to the involvement of medial rectus and lateral rectus muscles as suggested by MRI we anticipated the occurrence of oculocardiac reflex intra-operatively which was managed successfully by vigilant monitoring and timely pro-paracaine instillation. ${ }^{8}$ No prophylactic or intraoperative atropine was given for the fear of worsening of tricuspid regurgitation due to atropine induced tachycardia. Although our patient was clinically diagnosed as anophthalmic, imaging and surgical exploration showed the presence of a rudimentary eyeball and hence a final diagnosis of microphthalmia was made intra-operatively. At the end of the procedure, patient was reversed uneventfully.

The main concerns in the post-operative period for such cases are the risks of dysrhythmias and thromboembolic events and sometimes worsening of the shunt. Hence, the patients have to be nursed under constant observation with special attention given to alleviation of pain and maintaining hemodynamic stability. To achieve these goals, in the post-operative period, the patient was shifted to the high dependency unit (HDU) for two-days. On the third post-operative day the patient was discharged in stable condition as of pre-operative status, and was followed-up regularly as per schedule.

\section{CONCLUSION}

Congenital ophthalmopathy may be associated with multisystem disorders that may or may not be clinically evident. Detailed preoperative workup of the patient helps to anticipate, understand and manage the intra-operative complications that may arise during the course of surgery. It is therefore important to know the clinical associations of the congenital disorders and their anesthetic implications even in seemingly uncomplicated cases. Anesthesiologists must tailor the type and mode of anaesthesia accordingly. The main concerns of hemodynamic stability and normocarbia should be addressed in all such cases with pre-existing cardiac findings along with vigilant post-operative observation.

\section{CONSENT}

The patient has provided written permission for publication of the case details.

\section{CONFLICTS OF INTEREST}

The authors declare that they have no conflicts of interest.

\section{REFERENCES}

1. Morrison D, Fitzpatrick D, Hanson I, et al. National study of microphthalmia, anophthalmia and coloboma in scotland: Investigation of genetic aetiology. J Med Genet. 2002; 39: 16-22.

2. Campbell H, Holmes E, Mac Donald S, Morisson D, Jones I. A capture recapture model to estimate prevalence of children born in scotland with developmental eye defects. J Cancer Epidemiol Prev. 2002; 7: 21-28.

3. Kallen B, Robert E, Harris J. The descriptive epidemiology of anophthalmia and microphthalmia. Int J Epidemiol. 1996; 25: 10091116.

4. Aliyu I, Gambo S, Igoche PD. Congenital bilateral anophthalmia: A case report and review of literature. Sudan Medical Monitor . 2015; 10(2): 69-71. doi: 10.4103/1858-5000.160954

5. Shaw GM, Carmichael SL, Yang W, Harris JA, Finnell RH, Lammer EJ. Epidemiologic characteristics of anophthalmia and bilateral microphthalmia among 2.5 million births in California, 1989-1997. Am J Med Genet A. 2005; 137(1): 36-40. doi: 10.1002/ ajmg.a.30840

6. Krastinova D, Kelly MB, Mihaylova M. Surgical management of the anophthalmic orbit, part 1: Congenital. Plast Reconstr Surg. 2001; 108(4): 817-826.

7. Mazzoli RA, Raymond WR $4^{\text {th }}$, Ainbinder DJ, Hansen EA. Use of self-expanding, hydrophilic osmotic expanders (hydrogel) in the reconstruction of congenital clinical anophthalmos. Curr Opin Ophthalmol. 2004; 15(5): 426-431.

8. Gupta L, Jain T. ANSHI method: A new approach for oculocardiac reflex prevention. Anaesth Intensive Care Pain. 2018; 1(1): 1-1. 